\title{
Drifting pulsating structures generated during tearing and coalescence processes in a flare current sheet
}

\author{
M. Karlický and M. Bárta
}

\begin{abstract}
Astronomical Institute of the Academy of Sciences of the Czech Republic, 25165 Ondřejov, Czech Republic e-mail: [karlicky; barta]@asu.cas.cz
\end{abstract}

Received 6 July 2006 / Accepted 4 December 2006

\section{ABSTRACT}

\begin{abstract}
Aims. Based on particle-in-cell simulations, drifting pulsating structures are interpreted as the radio emission generated during tearing and coalescence processes in the current sheet of a flare.

Methods. A 2.5D particle-in-cell electromagnetic relativistic code was used considering two versions of the model: a) the model with one current sheet with free boundary conditions and b) the model with two current sheets and periodic boundary conditions. The dispersion diagrams of electromagnetic waves we then constructed using the 2D Fourier transform.

Results. It is found that electrons are accelerated most efficiently in the region near the x-point of the magnetic field structure in the phase at the end of tearing process and at the beginning of plasmoid coalescence. The most energetic electrons are distributed mainly along the $x$-lines of the magnetic field structure. During these processes, Langmuir waves are generated, along with the electromagnetic (radio) ones. It looks as these electromagnetic waves can be observed mainly on the second harmonics of the plasma frequencies corresponding to the plasma densities in the external parts of the plasmoids.
\end{abstract}

Key words. Sun: flares - Sun: radio radiation - acceleration of particles

\section{Introduction}

The papers by Kliem et al. (2000), Khan et al. (2002), and Karlický (2004) found a relationship between the drifting pulsating structures (DPSs) observed in radio waves in the decimetric range and ejection of X-ray plasmoids (e.g. Ohyama \& Shibata 1998). Based on a 2D MHD numerical modelling of the solar flare reconnection, Kliem et al. (2000) proposed that the DPSs are generated during a bursting regime of the magnetic-field reconnection when interacting plasmoids (magnetic islands) are formed. It was proposed that electrons are accelerated during these processes and trapped in the plasmoids. Furthermore, it was suggested that these accelerated electrons generate individual pulses of the observed DPSs. The global frequency drift of DPS was explained by a motion of the plasmoid in the density gradient of the solar atmosphere. In Karlický (2004) we used the concept of the fractal reconnection proposed by Shibata \& Tanuma (2001) and considered several plasmoids to explain simultaneous observation of several DPSs. An example of DPS observed at the beginning of the 2005 June 14 C4.2 flare is shown in Fig. 1.

Up to now, all models explaining the DPSs have been based on the MHD modelling, which gives us no information about kinetic and radio emission processes. To overcome this difficulty in the present paper, we simulate the tearing and coalescence processes in the current sheet using the 2.5D particle-in-cell (PIC) model. In the classical model of two-ribbon flares and in our previous studies of DPSs, only one current sheet was considered (Priest \& Forbes 2000; Ohyama \& Shibata 1998; Shibata 1999; Kliem et al. 2000; Karlický 2004). But there are still debates based on the magnetospheric observations (Liu et al. 2000) that the flare current sheet can be structured and that some flare models assume many current sheets (Turkmani et al. 2006).

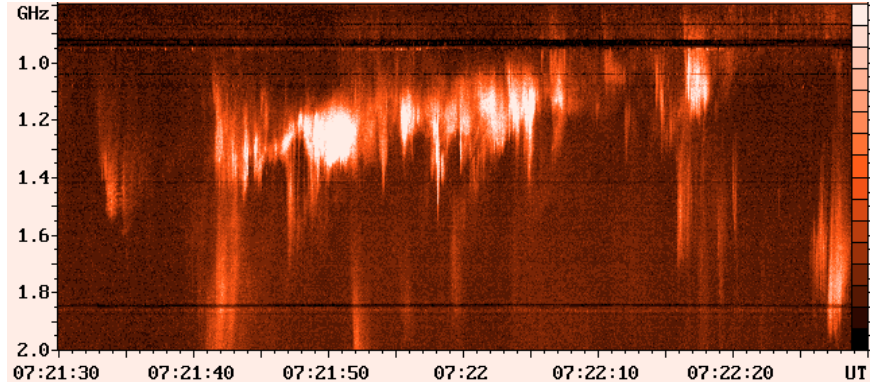

Fig. 1. The $0.8-2.0 \mathrm{GHz}$ radio spectrum showing the drifting pulsating structure, observed by the Ondřejov radiospectrograph (Jiřička et al. 1993) at 07:21:30-07:22:30 UT, on June 14, 2005.

Therefore in this paper we consider two versions of the model: the model with one current sheet with free boundary conditions and the model with two current sheets with periodic boundary conditions. Finally, dispersion diagrams of generated waves have been constructed.

\section{Model}

To simulate the processes under study, we used a 2.5D (2D3V 2 spatial and 3 velocity components) fully relativistic electromagnetic particle-in-cell code (Saito \& Sakai 2004). The system size is $L_{x}=2000 \Delta$ and $L_{y}=600 \Delta$, where $\Delta(=1)$ is a grid size. As mentioned in the introduction, we considered two versions of the model:

Model 1 with one current sheet with free boundary conditions: The 2D current sheet with the guiding magnetic field $B_{z}$ is initiated along the line $y=300 \Delta$. The half-width of the current 
sheet is $10 \Delta$. The initial magnetic field is

$$
\begin{array}{r}
\boldsymbol{B} \equiv\left(B_{x}, B_{y}, B_{z}\right), \\
B_{x}=-B_{0} \text { for } y<290 \Delta, \\
B_{x}=(y-300) B_{0} / 10 \text { for } 290 \Delta \leq y \leq 310 \Delta, \\
B_{x}=B_{0} \text { for } y>310 \Delta, \\
B_{y}=0, B_{z}=B_{0} .
\end{array}
$$

Model 2 with two current sheets with periodic boundary conditions: two 2D current sheets with the guiding magnetic field $B_{z}$ are initiated along the lines $y=150 \Delta$ and $y=450 \Delta$. The halfwidth of both the current sheets is $10 \Delta$. The initial magnetic field is

$$
\begin{array}{r}
\boldsymbol{B} \equiv\left(B_{x}, B_{y}, B_{z}\right), \\
B_{x}=-B_{0} \text { for } y<140 \Delta, \\
B_{x}=(y-150) B_{0} / 10 \text { for } 140 \Delta \leq y \leq 160 \Delta, \\
B_{x}=B_{0} \text { for } 160 \Delta<y<440 \Delta, \\
B_{x}=-(y-450) B_{0} / 10 \text { for } 440 \Delta \leq y \leq 460 \Delta, \\
B_{x}=-B_{0} \text { for } y>460 \Delta, \\
B_{y}=0, B_{z}=B_{0} .
\end{array}
$$

The electron-proton plasma with the proton-electron mass ratio $m_{\mathrm{p}} / m_{\mathrm{e}}=16$ is considered in both these models. It is unrealistic and taken to shorten computations. Nevertheless, the electron mass is low enough to separate the dynamics of electrons and protons well. In each numerical cell located out of the current sheet we initiated $n_{0}=60$ electrons and $n_{0}=60$ protons. The initial number density is enhanced in the current sheets just to keep the pressure equilibrium in the current sheet. The electron thermal velocity is taken to be the same in the whole numerical box as $v_{\mathrm{Te}}=0.1 c$, where $c$ is the speed of light. The corresponding electron temperature is $T=59.5 \mathrm{MK}$, i.e. higher than expected at the beginning of the impulsive flare phase, but not out of the range of temperatures measured in flares (Hoyng et al. 1981). The main reason for such high temperature is to shorten long computations, i.e. the growth rate of the collisionless tearing-mode instability is proportional to the thermal velocity (Drake \& Lee 1977). The temperature of protons was chosen to be the same as for electrons.

In the current sheets, the appropriate electric current densities are initiated. The Debye length corresponds to grid size $\Delta$. The plasma beta parameter and the ratio of the electroncyclotron and electron-plasma frequencies in the region outside of the current sheets are chosen as $\beta=0.2$ and $\omega_{\mathrm{ce}} / \omega_{\mathrm{pe}}=0.4$, respectively.

All computations were performed on the parallel computer OCAS (Ondřejov Cluster for Astrophysical Simulations), see http://wave.asu.cas.cz/ocas/.

\section{Results}

\subsection{Model 1}

The time evolution of the current sheet under the influence of the tearing and coalescence instabilities in four instants is shown in Figs. 2 and 3. As seen there, the magnetic islands with enhanced densities (plasmoids) are formed and then they coalesce at about $\omega_{\mathrm{pe}} t=3000$ into one single plasmoid. The density structure of the plasmoids is rather complicated, which indicates that the plasmoids are not in equilibrium.

The distribution functions of electrons, as well as protons, evolve during these processes. We found their most distinct
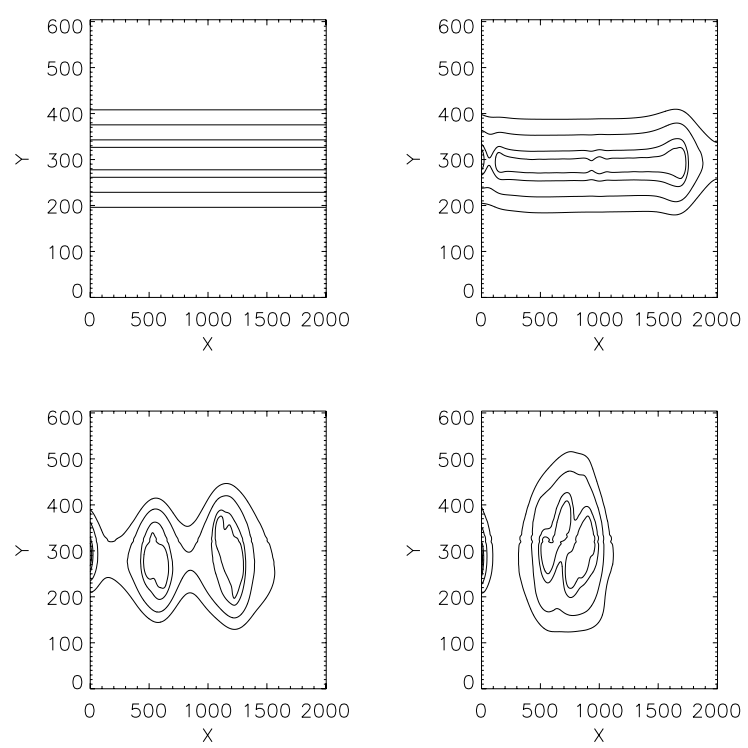

Fig. 2. Model 1: magnetic field lines projected onto the $x-y$ plane at four different times: the initial state (upper left), at $\omega_{\mathrm{pe}} t=1000$ (upper right), at $\omega_{\mathrm{pe}} t=2000$ (bottom left), and $\omega_{\mathrm{pe}} t=3000$ (bottom right).
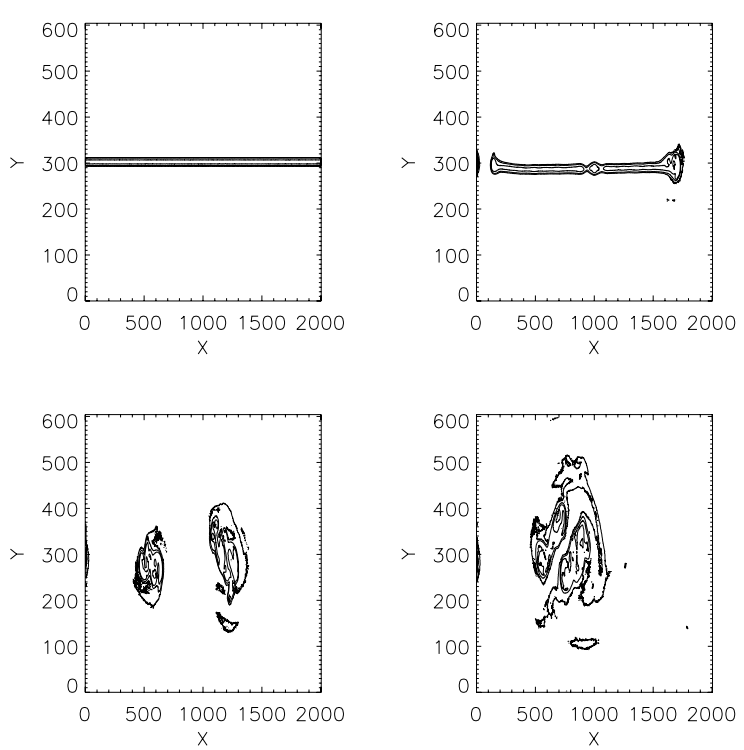

Fig. 3. Model 1: electron plasma density contours (levels 100, 150, and 300 electrons per cell; the density in the region outside of the current sheet is $n_{0}=60$ electrons per cell) at four different times: the initial state (upper left), at $\omega_{\mathrm{pe}} t=1000$ (upper right), at $\omega_{\mathrm{pe}} t=2000$ (bottom left), and $\omega_{\mathrm{pe}} t=3000$ (bottom right).

changes in the space close to the $x$-point of the magnetic field structure (i.e. at the position $x=800 \Delta$ and $y=300 \Delta$ ) in the time interval $\omega_{\mathrm{pe}} t=1600-2200$ (Fig. 4). At this time interval, which corresponds to the ending phase of tearing processes of the current sheet and to the beginning phase of coalescence of plasmoids, a distinct asymmetric non-thermal tail in the $v_{z}$ velocity component can be seen. Before and after this time interval and in other locations, the distribution functions have more or less Maxwellian (i.e. thermal) forms. The plasma temperature increases everywhere during the evolution. On the other hand, it is interesting to see that energetic electrons $(|v|>0.3 \mathrm{c})$ are distributed mainly along the $x$-lines of the magnetic field structure and their continuations (Fig. 5). 

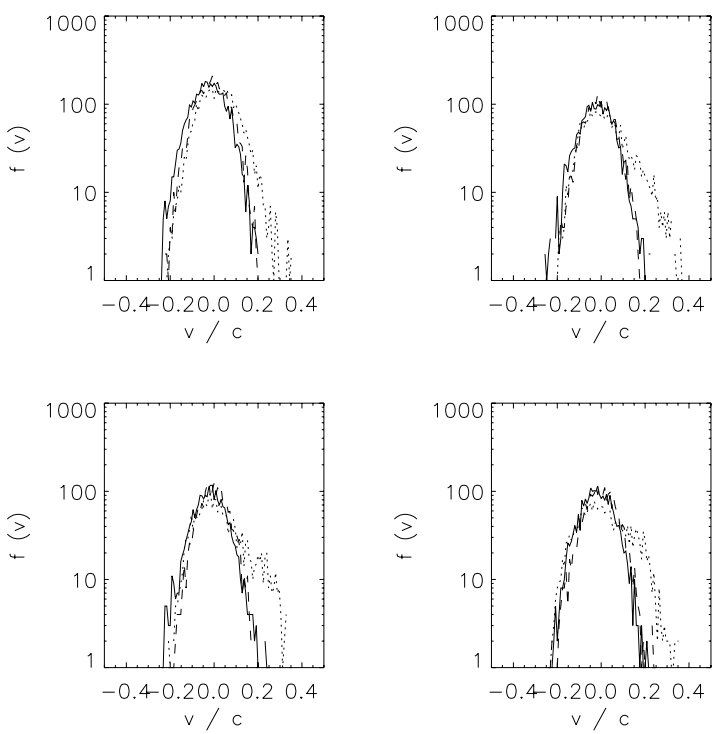

Fig. 4. Model 1: electron distribution functions (full line means $f\left(v_{x} / c\right)$, dashed line $f\left(v_{y} / c\right)$, and dotted line $f\left(v_{z} / c\right)$ ) in the circular region with the radius $r=70 \Delta$ and the centrum at $x=800 \Delta$ and $y=300 \Delta$ at four different times: at $\omega_{\mathrm{pe}} t=1600$ (upper left), at $\omega_{\mathrm{pe}} t=1800$ (upper right), at $\omega_{\mathrm{pe}} t=2000$ bottom left), and at $\omega_{\mathrm{pe}} t=2200$ (bottom right).

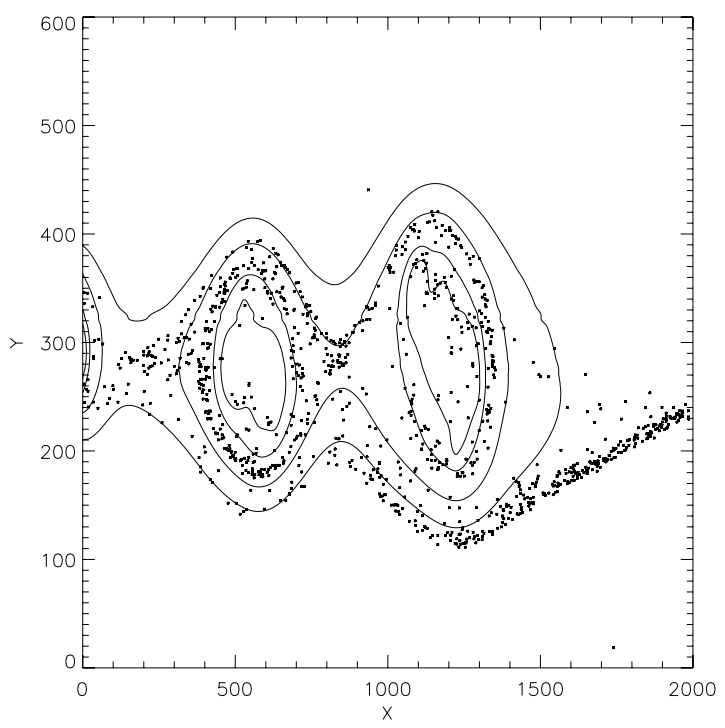

Fig. 5. Model 1: the distribution of energetic electrons $(|v|>0.3 \mathrm{c})$ in the $x-y$ plane.

To know what kind of plasma waves are generated during these processes, we computed dispersion diagrams of the electric field components $E_{x}, E_{y}$, and $E_{z}$ recorded along the line $y=300 \Delta$ in the numerical box throughout the whole particlein-cell simulation. We used the 2D Fourier transform of these components in two spatial intervals $x=(250 \Delta-250+512 \Delta)$ and $x=(600 \Delta-600+512 \Delta)$, and in the time interval $\omega_{\mathrm{pe}} t=$ (1900-2104.8) (4096 time steps). These intervals correspond to plasma processes in the left plasmoid and in the region between two interacting plasmoids (for locations, see Figs. 2, 3, and 5) at the phase of the non-thermal electron acceleration. The dispersion diagrams of the electric field components $E_{x}$ and $E_{z}$ for the spatial interval $x=(600 \Delta-600+512 \Delta)$ are shown in Fig. 6. (The dispersion diagram for the electric field component $E_{y}$ is similar to that of $E_{x}$.) While the dispersion diagram of $E_{x}$ shows plasma (Langmuir) waves (probably in a turbulent state)
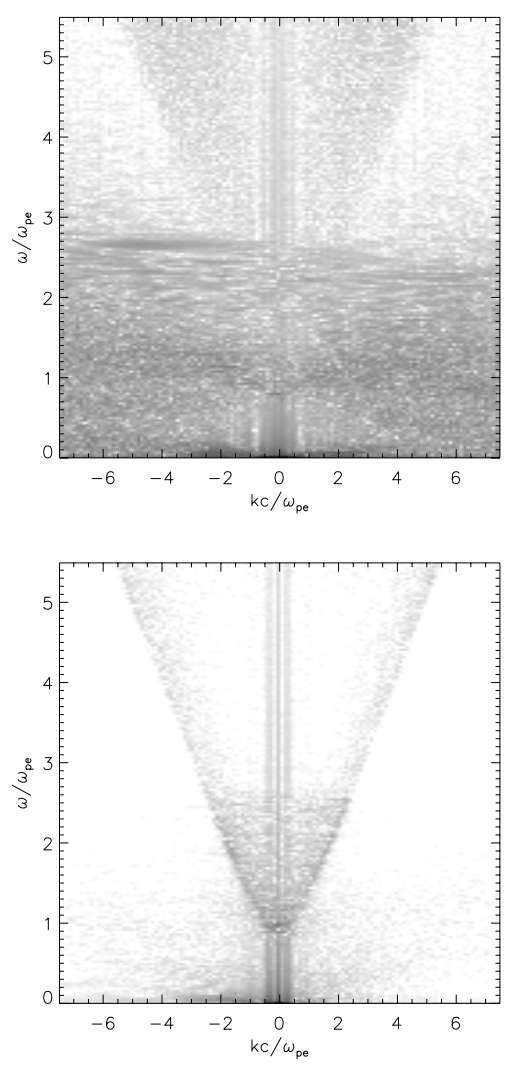

Fig. 6. Model 1: dispersion diagrams obtained by the 2D Fourier transform of the electric field components $E_{x}$ (upper) and $E_{z}$ (bottom) computed along the line with $x=(600 \Delta-600+512 \Delta), y=300 \Delta$, in the time interval $\omega_{\mathrm{pe}} t=(1900-2104.8)$, i.e. 4096 time steps. Darker spots mean intense waves.

in the interval of plasma frequencies from $0.9-2.7 \omega_{\text {pe }}$, the dispersion diagram for $E_{z}$ shows the electromagnetic waves in the same frequency range. The plasma frequency $\omega_{\text {pe }}$ corresponds to the initial density in the region outside of the current sheet, i.e. $n_{0}=60$ electrons per cell. The density in the system at the times $\omega_{\mathrm{pe}} t=(1900-2104.8)$ varies from $n=0.83 n_{0}$ in the region between the plasmoids to $n=7.2 n_{0}$ in the central parts of plasmoids. The energetic electrons can be found in the whole interval of these plasma densities. But, most of them are at the locations with the densities $n=0.83-2.1 n_{0}$ (external parts of the plasmoids).

\subsection{Model 2}

The time evolution of the system with two current sheets is shown for four instants $\left(\omega_{\mathrm{pe}} t=0, \omega_{\mathrm{pe}} t=2000, \omega_{\mathrm{pe}} t=3500\right.$, and $\omega_{\mathrm{pe}} t=5000$ ) in Figs. 7 and 8. As seen there, the bottom current sheet evolves more rapidly than the upper one. During this process electrons are accelerated and heated as shown in Fig. 9, where an evolution of the electron distribution function in full computational box is presented. The hardest power-law index of the distribution function reached in these computations is -5.8 .

As in Model 1, the most effective acceleration of electrons is found in the space close to the $x$-point of the magnetic field structure (i.e. at about the position $x=1000 \Delta$ and $y=150 \Delta$ ) in the time interval $\omega_{\mathrm{pe}} t=1500-3000$ (Fig. 10). At this time interval, which corresponds to the ending phase of tearing processes in the current sheet and to the beginning phase of coalescence of plasmoids, a distinct asymmetric non-thermal tail in the $v_{z}$ 

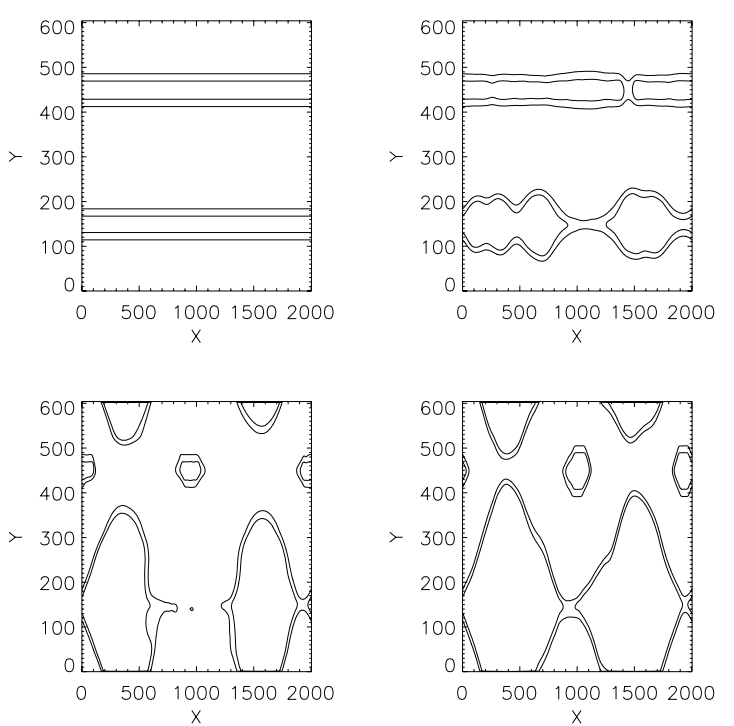

Fig. 7. Model 2: magnetic field lines projected onto the $x-y$ plane at four different times: at the initial state (upper left), at $\omega_{\text {pe }} t=2000$ (upper right), at $\omega_{\mathrm{pe}} t=3500$ (bottom left), and $\omega_{\mathrm{pe}} t=5000$ (bottom right).
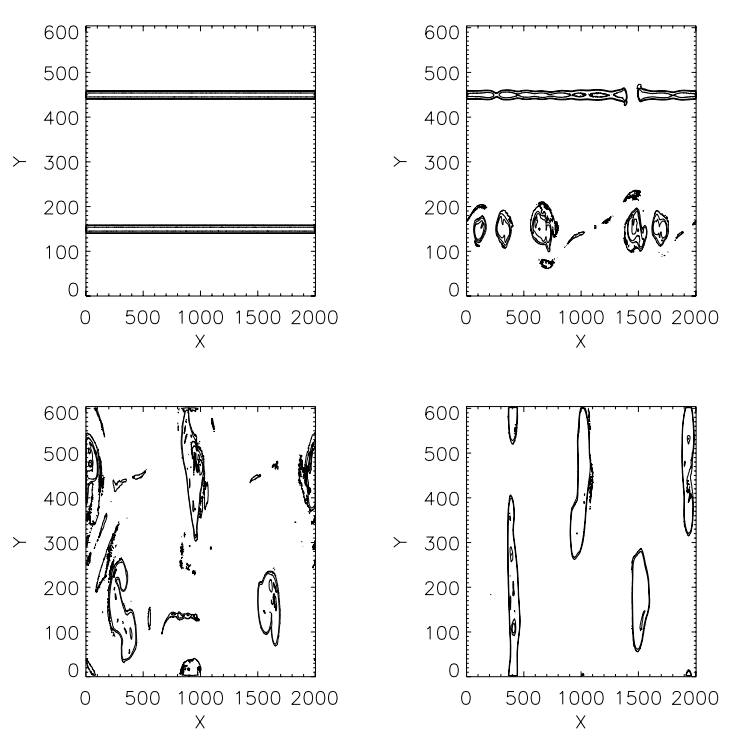

Fig. 8. Model 2: electron plasma density contours (levels 100, 150, and 300 electrons per cell; the density in the region outside of the current sheets is $n_{0}=60$ electrons per cell) at four different times: the initial state (upper left), at $\omega_{\mathrm{pe}} t=2000$ (upper right), at $\omega_{\mathrm{pe}} t=3500$ (bottom left), and $\omega_{\mathrm{pe}} t=5000$ (bottom right).

velocity component can be seen. Before and after this time interval and in other locations, the distribution functions have more or less Maxwellian forms. The plasma temperature increases everywhere during the evolution. On the other hand, the energetic electrons $(|v|>0.3 \mathrm{c})$ are distributed along the $x$-lines of the magnetic field structure, as well as in the plasmoids (Fig. 11).

In this model, as in Model 1, we computed dispersion diagrams of the electric-field components $E_{x}, E_{y}$, and $E_{z}$ recorded along the line $y=300 \Delta$ in the numerical box throughout the whole particle-in-cell simulation. But in this model the electric field components were recorded outside of both the current sheets. We considered the spatial and temporal intervals $x=(750 \Delta-750+512 \Delta)$ and $\omega_{\mathrm{pe}} t=(1900-2104.8)$ (4096 time steps), respectively. The dispersion diagrams of the electric field components $E_{y}$ and $E_{z}$ are shown in Fig. 12. (The dispersion
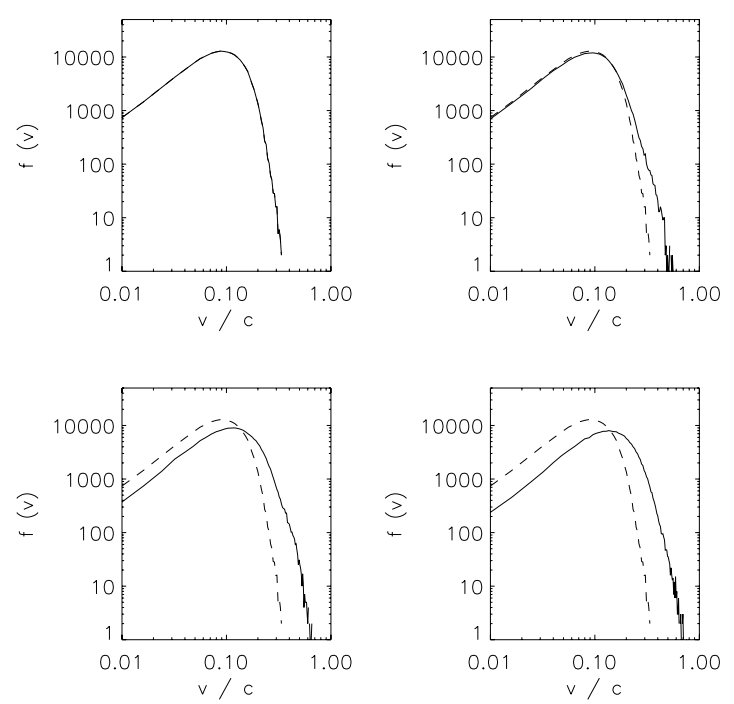

Fig. 9. Model 2: the electron distribution function $f(|v| / c)$ in the logarithm scales in the full computational box at four different times: $\omega_{\mathrm{pe}} t=0$ (upper left), at $\omega_{\mathrm{pe}} t=2000$ (upper right), at $\omega_{\mathrm{pe}} t=3500$ (bottom left), and at $\omega_{\mathrm{pe}} t=5000$ (bottom right). The dashed line means the distribution function at the initial state.
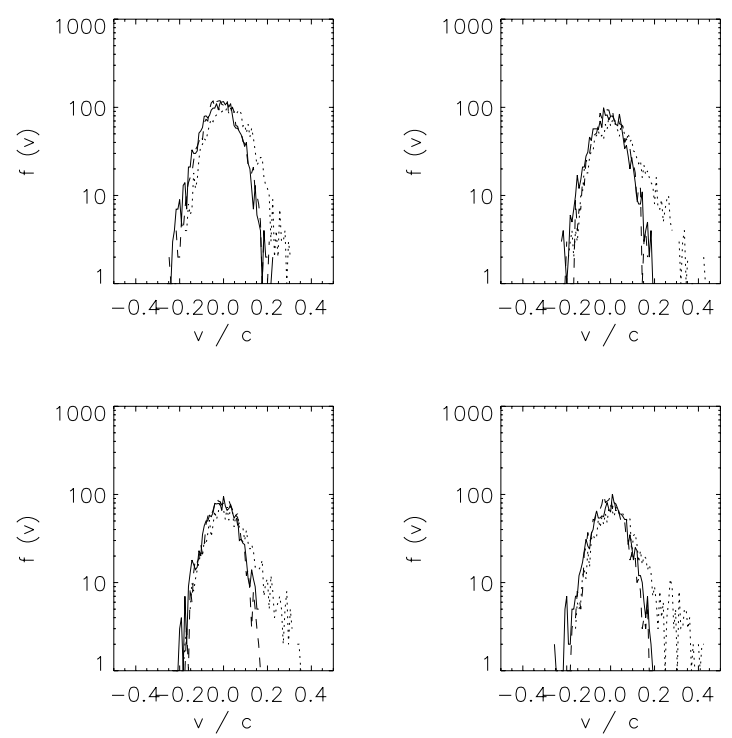

Fig. 10. Model 2: electron distribution functions (full line means $f\left(v_{x} / c\right)$, dashed line $f\left(v_{y} / c\right)$, and dotted line $f\left(v_{z} / c\right)$ ) in the circular region with the radius $r=70 \Delta$ and the centrum at $x=1000 \Delta$ and $y=150 \Delta$ at four times: at $\omega_{\mathrm{pe}} t=1500$ (upper left), at $\omega_{\mathrm{pe}} t=2000$ (upper right), at $\omega_{\mathrm{pe}} t=2500$ (bottom left), and at $\omega_{\mathrm{pe}} t=3000$ (bottom right).

diagram for the electric field component $E_{x}$ is similar to that of $E_{y}$.) In the dispersion diagram of $E_{y}$, we can see a branch of the plasma (Langmuir) waves at the frequency $\omega \sim \omega_{\text {pe }}$. The energy of these Langmuir waves is low because they are in the region outside of the current sheets, i.e. in the region where there are no energetic electrons, see Fig. 11. On the other hand, in both of the dispersion diagrams, we can see wave energy enhancements in two frequency intervals $\left(1.2-1.7 \omega_{\text {pe }}\right.$ and 2.3-3.3 $\left.\omega_{\text {pe }}\right)$ along the electromagnetic wave branch (the hyperbola curve in the dispersion diagrams). The wave energy in the frequency interval of 2.3-3.3 $\omega_{\mathrm{pe}}$ is about two orders of magnitude stronger than in the 1.2-1.7 $\omega_{\text {pe }}$ interval. A detailed analysis shows the energetic electrons are distributed in the current sheets where the 


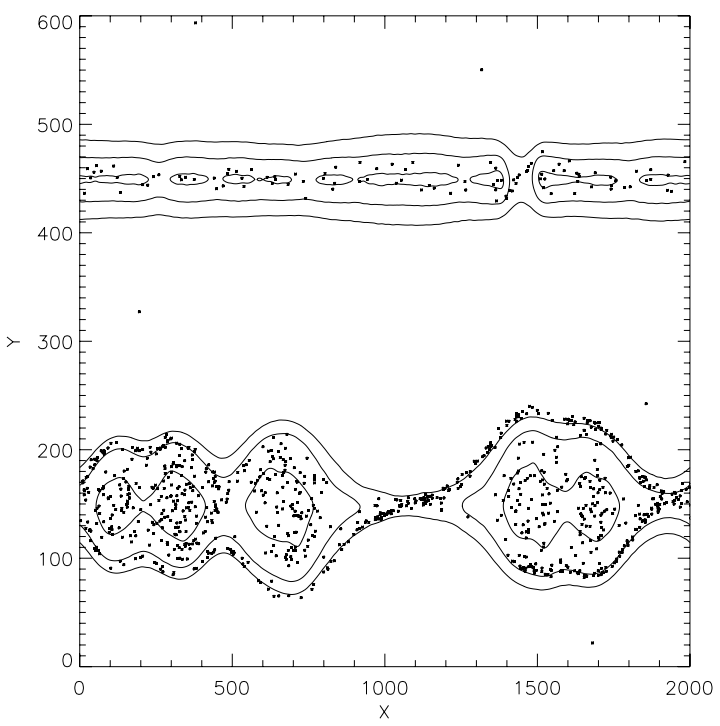

Fig. 11. Model 2: the distribution of energetic electrons $(|v|>0.3 \mathrm{c})$ in the $x-y$ plane.

densities are $n=0.83-8.3 n_{0}$ (where $n_{0}$ is 60 electrons per cell), but most of them are at the locations with the densities from $n=0.83-2.1 n_{0}$ (external parts of the plasmoids).

\section{Discussion and conclusions}

The results of the present modelling confirm the suggestions made by Kliem et al. (2000), Khan et al. (2002), and Karlický (2004).

In two versions of the PIC model it was found that electrons are the most effectively accelerated in the region near to the main $x$-point of the magnetic field structure in the phase at the end of tearing processes and at the beginning of coalescence of plasmoids. The most energetic electrons were localized mainly along the $x$-lines of the magnetic field structure.

The results obtained in Model 2 are very similar to those presented by Drake et al. (2005). As in their paper, we found that the most energetic particles are localized along the $x$-lines of a magnetic-field structure. But, some energetic electrons are also found inside the main plasmoids. The detailed analysis shows that inside these main plasmoids there are further $x$-points (see Fig. 11) where the electrons are accelerated as in the main $x$ points. The accelerated electrons move along separatrixes and also drift together with magnetic field lines under the influence of an inductive electric field. During this pinch process of plasmoids, the electrons are accelerated more.

In Model 2 the bottom current sheet evolves faster than the upper one (Fig. 11). In our computations the tearing process starts without any trigger. It starts from the numerical noise level; therefore, in the current sheets with random initial thermal particle distributions, the starting phase does not have to be the same. In the later phase, the close current sheets interact due to plasma flows and thus producing the asymmetry in their development.

To shorten our computations, we used a high value of the initial electron thermal velocity $\left(v_{\mathrm{Te}}=0.1 c\right)$ in our model. We also made additional computations with $v_{\mathrm{Te}}=0.05 c$. The results are qualitatively the same. Based on these additional computations and in accordance with Drake et al. (2005), we can conclude that the energies of accelerated electrons increase with an increase in the initial temperature, e.g. due to a faster tearing process. But generally the maximum energy also depends on the system size;
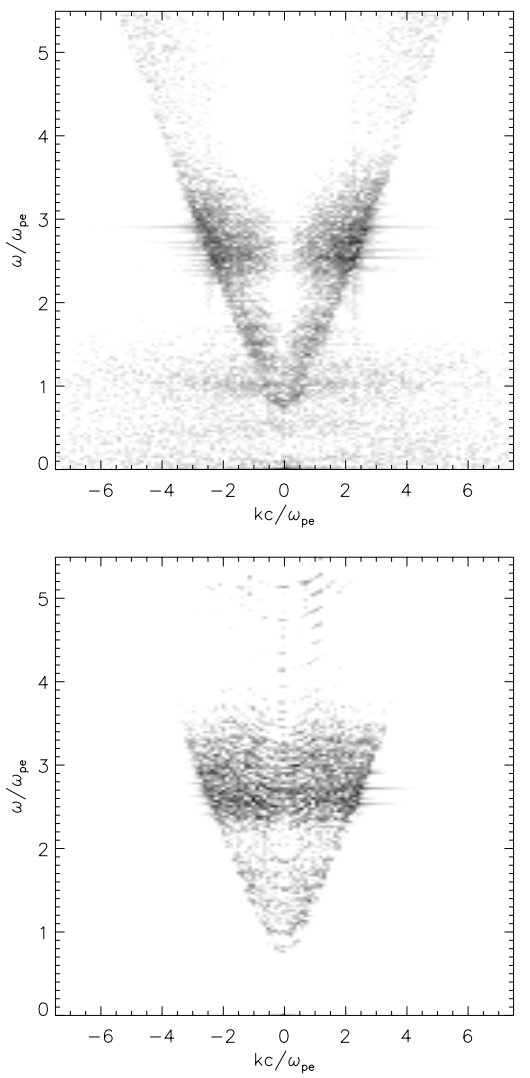

Fig. 12. Model 2: dispersion diagrams obtained by the 2D Fourier transform of the electric field components $E_{y}$ (upper) and $E_{z}$ (bottom) computed along the line with $x=(750 \Delta-750+512 \Delta), y=300 \Delta$ in the time interval $\omega_{\text {pe }} t=(1900-2104.8)$, i.e. 4096 time steps. Darker spots mean intense waves.

namely, in large systems with many $x$-points due to multiple interactions, the electrons can be accelerated to very high energies.

The dispersion diagrams of generated waves were computed in both models. In Model 1 these diagrams were obtained in the evolving current sheet, i.e. in the plasmoids and in the region between them. In the positions due to fast spatial and temporal density changes, the dispersion diagrams are complex, especially for the electric field components in the $x-y$ plane. Nevertheless, they show that both the Langmuir and electromagnetic waves are produced in the limited frequency range. On the other hand, the dispersion diagrams in Model 2 were obtained in the region out of the current sheets where the plasma density was constant $\left(=n_{0}\right)$. Thus, the dispersion diagrams are less complex than those in Model 1 and more informative. Besides the Langmuir waves at the thermal level (no energetic electrons at the region) on local plasma frequency, the electromagnetic waves produced at "distant" current sheets can be seen on these diagrams. They consisted of two bands (1.2-1.7 $\omega_{\text {pe }}$ and 2.3-3.3 $\left.\omega_{\text {pe }}\right)$ in a harmonic relation. It looks as if these bands are the plasma emission of accelerated electrons in the fundamental and harmonic bands of plasma frequencies in the plasmoids. It was found that the emission in the harmonic band is about 100 times stronger than in the fundamental one. In agreement with the analysis of positions of energetic electrons and densities at these locations, it looks as if the emission is produced in the external parts of the plasmoids. The plasma densities, as well as the plasma frequencies, are smaller at these positions than in the central parts of the plasmoids. 
The limited frequency range of generated waves explains the limited bandwidth of observed DPSs (e.g. Fig. 1). Sometimes, depending on the magnetic field structure, some energetic electrons can escape from the acceleration region and plasmoids, and thus these electrons can generate additional type III-like bursts (see Fig. 1, the radio features outside the main body of DPS).

We plan to model the effects of the global frequency drift of DPS and pulsating character of this burst using a combined MHD and kinetic model where the density gradient of the solar atmosphere will be included.

Acknowledgements. The authors thank the referee for constructive comments. This research was supported by the Centre for Theoretical Astrophysics and by Grants IAA3003202 of the Academy of Sciences and Grant 205/04/0358 of the Grant Agency of the Czech Republic. M.K. thanks Prof. J. I. Sakai and Dr. S. Saito for many useful discussions concerning numerical modelling.

\section{References}

Drake, J. F., Shay, M. A., Thongthai, W., \& Swisdak, M. 2005, Phys. Rev. Lett., 94 (9), 095001

Drake, J. F., \& Lee, Y. C. 1977, Phys. Fluids, 20, 1341

Hoyng, P., Duijveman, A., Machado, M. E., et al. 1981, ApJ, 246, 155

Jiřička, K., Karlický, M., Kepka, O., \& Tlamicha, A. 1993, Sol. Phys., 147, 203

Karlický, M. 2004, A\&A, 417, 325

Khan, M., Vilmer, N., Saint-Hilaire, P., \& Benz, A. O. 2002, A\&A, 388, 363

Kliem, B., Karlický, M., \& Benz, A. O. 2000, A\&A, 360, 715

Lui, A. T. Y., Chapman, S. C., Liou, K., et al. 2000, Geophys. Res. Lett., 27, 911 Ohyama, M., \& Shibata, K. 1998, ApJ, 499, 934

Priest, E. R., \& Forbes, T. 2000, Magnetic Reconnection: MHD Theory and Applications (Cambridge, UK: Cambridge Univ. Press)

Saito, S., \& Sakai, J. I. 2004, ApJ, 616, L179

Shibata, K. 1999, Ap\&SS, 264, 129

Shibata, K., \& Tanuma, S. 2001, Earth Planets Space, 53, 473

Turkmani, R., Cargill, P. J., Galsgaard, K., Vlahos, L., \& Isliker, H. 2006, A\&A, 449,749 